\title{
Incertitude et action publique. Définition des risques, production des savoirs et cadrage des controverses
}

\author{
Sébastien Chailleux-Centre Emile Durkheim
}

\section{Introduction}

Les hydrocarbures non conventionnels, dont le gaz de schiste fait partie, deviennent une industrie mature dans la seconde moitié des années 2000 aux États-Unis ; mais le déploiement de celui-ci hors des frontières américaines demeure difficile puisqu'à la fois les configurations légales et politiques sont différentes ${ }^{1}$ mais aussi, pour le point qui nous intéresse ici, l'incertitude, qu'elle soit scientifique, économique, sociale ou politique, apparaît plus élevée. Elle est comprise comme une situation d'insuffisance des connaissances scientifiques, mais aussi d'imprévisibilité et de complexité des effets des actions engagées. Si les données géologiques américaines sont assez précises au sujet du gaz de schiste, ce n'est pas le cas pour les sous-sols français et québécois. Plus encore, les données à propos de la fracturation hydraulique, principale technique utilisée pour extraire le gaz et les huiles de schiste, sont encore controversées. Enfin, certains éléments sur les effets de l'industrie sur l'environnement, l'économie, les populations locales et le système légal manquent. Ainsi, lorsque le gaz de schiste devient un problème public en France et au Québec en 2010, les décideurs politiques doivent gérer un niveau élevé d'incertitude. S'ils s'appuient alors sur les connaissances existantes, ils mettent également en œuvre des dispositifs (étude d'impact, audition d'experts) afin de la réduire et de gérer la crise qui lui est liée. La controverse englobe des enjeux innombrables, cet article se limite à analyser l'incertitude qui concerne le niveau de ressources potentielles, la maîtrise technique, la pollution des eaux souterraines, la rentabilité économique et l'acceptabilité sociale. II vise à démontrer comment les acteurs luttent pour imposer ou réduire le niveau d'incertitude afin de défendre un agenda particulier, et de quelle façon les décideurs politiques, élus et fonctionnaires, gèrent cette incertitude socialement produite en faisant appel à des experts et en cadrant diversement le problème.

Le croisement théorique de trois approches

Le problème du gaz de schiste en France et au Québec est en train d'émerger dans les sciences sociales, mais n'est souvent redevable que d'analyses segmentées. Certains chercheurs s'appuient plutôt sur la sociologie

\footnotetext{
${ }^{1}$ Pour de plus amples explications, voir CHAILLEUX, S., "Non au gaz de schiste! " Cadrages et débordements de la controverse sur les hydrocarbures non conventionnelles en France et au Québec, Thèse pour l'obtention du doctorat en science politique, Université de Bordeaux, 2015
} 
des mouvements sociaux ${ }^{2}$ et d'autres sur la sociologie de l'action publique ${ }^{3}$. La production et la circulation des savoirs a par ailleurs reçu moins d'attention ${ }^{4}$. Le présent article vise à l'inverse à prendre à bras le corps ces trois dimensions en y ajoutant une composante comparative. Cet article se propose de croiser trois approches de sociologie politique - la sociologie des problèmes publics et des risques, l'analyse des politiques publiques et la sociologie des sciences et de l'expertise - afin de montrer leur complémentarité pour saisir les enjeux de la production de savoir et de l'ignorance par et pour les décideurs politiques à travers l'exemple de la controverse sur le gaz de schiste en France et au Québec (Canada) $)^{5}$.Trois pistes fructueuses semblent ainsi pouvoir être explorées. .

D'abord, la sociologie des controverses et des risques ${ }^{6}$, et plus largement la sociologie des problèmes publics, a depuis longtemps démontré comment les innovations et les progrès technologiques produisaient de nouveaux problèmes ${ }^{7}$, et comment la "modernité " transformait nos relations avec une certitude "probabilistique $»^{8}$. Le risque est une variable importante de la décision publique et sa prise en charge interroge les pratiques démocratiques ${ }^{9}$. De nombreux chercheurs ont aussi montré de quelle(s) manière(s) un problème public est produit socialement ${ }^{10}$ par des acteurs à travers des processus tel que «naming,

\footnotetext{
${ }^{2}$ TERRAL P.-M., « La fronde contre le gaz de schiste : essai d'histoire immédiate d'une mobilisation éclair (2010-2011) ", Écologie \& politique, vol. 45 / 2, 2012, p. 185. BHERER L., DUFOUR P., ROTHMAYR ALLISON C., « Analyse comparée des mobilisations autour du développement du gaz de schiste au Québec, en France, aux États-Unis et en ColombieBritannique ", Université de Montréal - Centre de recherche sur les politiques et le développement social, 2013. FORTIN M.-J., FOURNIS Y., " Une participation conflictuelle : la trajectoire territoriale des mobilisations contre le gaz de schiste au Québec », Participations, vol. 13/1, 2015, p.199-144

${ }^{3}$ CHAILLEUX S, MOYSON S, "The French ban on hydraulic fracturing and the attempts to reverse it: Social mobilization, professional forums and coalition strategies". In Policy Debates on Hydraulic Fracturing: Comparing Coalition Politics in North America and Europe, dirigé par C. Weible, T. Heikkila, K. Ingold and M. Fischer. New-York: Palgrave Macmillan, 2016.

${ }^{4}$ MOLINATTI G., SIMMONEAU L,. “A Socioenvironmental Shale Gas Controversy: Scientists' Public Communications, Social Responsibility and Collective Versus Individual Positions". Science Communication 37/2, 2016, p.190-216. GENDRON C., "Une science pacificatrice au service de l'acceptabilité sociale ? Le cas des gaz de schiste au Québec », Ethique publique, vol. 18 / 1, 2016

${ }^{5}$ Une controverse est ici définie comme une situation de désaccords à la fois scientifiques et techniques, ainsi que sociaux et politiques, qui oppose différents acteurs dans plusieurs arènes (scientifique, politique, médiatique ou publique).

${ }^{6}$ LEMIEUX C., "À quoi sert l'analyse des controverses ? ", Mil neuf cent - Revue d'histoire intellectuelle, vol. 25 / 1, 2007, p. 191-212.

${ }^{7}$ BORRAZ O., Les politiques du risque, Paris, Presses de Sciences Po, 2008.

${ }^{8}$ BECK U., GIDDENS, LASH S., Reflexive modernity, Cambridge, Polity Press, 1994.

${ }^{9}$ CALLON M., LASCOUMES P., BARTHE Y., Agir dans un monde incertain - Essai sur la démocratie technique, Paris, Seuil, 2001. NEVEU C., " Démocratie participative et mouvements sociaux : entre domestication et ensauvagement ? ", Participations, vol. 1 / 1, 2011, p. 186. BLATRIX C., BLONDIAUX L., FOURNIAU J.-M., [et al.], Le débat public : une expérience française de démocratie participative, Paris, La Découverte, 2007.

${ }^{10}$ GUSFIELD J., La culture des problèmes publics : L'alcool au volant : la production d'un ordre symbolique, Paris, Economica, 2008.
} 
claiming, blaming ${ }^{11}$. Un problème ne devient donc public que s'il est étiqueté comme tel, et il en est de même pour le risque et l'incertitude.

L'article s'appuie ensuite sur l'analyse interprétative des politiques publiques, notamment celle qui analyse les opérations de framing $^{12}$ afin de montrer comment le travail d'argumentation ${ }^{13}$ produit un certain cadre au sein duquel la controverse - et ses incertitudes - est débattue. L'idée défendue, en suivant l'exemple de S. Jasanoff ${ }^{14}$, est qu'un cadrage officiel, sociopolitique et sociocognitif, d'une controverse est généré au niveau institutionnel. Celui-ci est à la fois produit et producteur de dispositifs institutionnels et parlementaires et de sélection des expertises légitimes. Ce cadrage, défini comme une manière de regarder le monde à travers des réseaux d'inférences cognitives, est l'objet de la lutte entre les différents acteurs qui cherchent à imposer leur définition des enjeux. Ce travail narratif et cognitif canalise l'incertitude sur certains aspects et laisse de côté d'autres manques dans la connaissance, confirmant que ce n'est pas l'incertitude en elle-même qui pose problème mais son cadrage comme un problème politique. L'incertitude est ensuite gérée à travers différents dispositifs, ou instruments ${ }^{15}$, qui informent et définissent la manière dont elle est comprise.

Enfin, le dernier courant de recherche intégré à l'analyse est la sociologie de l'expertise ${ }^{16}$. Différents travaux ont souligné le besoin d'une sociologie politique des sciences faisant le pont entre les lieux de production de connaissances scientifiques, les institutions qui les régissent et les décisions politiques. ${ }^{17}$ L'article montre ainsi le caractère construit et situé des savoirs experts et les liens qu'ils entretiennent avec les structures qui

\footnotetext{
${ }^{11}$ FELSTINER W., ABEL R., SARAT A., « The Emergence and Transformation of Disputes: Naming, Blaming, Claiming ", Law \& Society Review, vol. 15 / 3-4, 1980, p. 631-654.

${ }^{12}$ GOFFMAN I., Frame analysis, Boston, Northeastern University Press, 1974.

${ }^{13}$ FORESTER J., FISCHER F., The argumentative turn in policy analysis and planning, London, Duke University Press, 1993. ZITTOUN P., La fabrique politique des politiques publiques: Une approche pragmatique de l'action publique, Paris, Presses de Sciences Po, 2014. FISCHER F., Democracy and Expertise: Reorienting Policy Inquiry, New York, Oxford University Press, 2009.

${ }^{14}$ JASANOFF S., States of Knowledge: The Co-production of Science and the Social Order, London, Routledge, 2004. JASANOFF S., Designs on Nature: Science and Democracy in Europe and the United States, Princeton University Press, 2005.

${ }^{15}$ LASCOUMES P. et LE GALES P., Gouverner par les instruments, Paris, Presses de la Fondation nationale des sciences politiques, 2004 ; HALPERN C., LASCOUMES P. et LE GALES P., L'instrumentation de l'action publique: controverses, résistances, effets, Paris, Presses de la Fondation nationale des sciences politiques, 2014.

${ }^{16}$ COLLINS H., EVANS R., op. cit.. WYNNE B., " Seasick on the Third Wave? Subverting the Hegemony of Propositionalism: Response to Collins \& Evans (2002) ", Social Studies of Science, vol. 33 / 3, 2003, p. 401-417. JASANOFF S., "Breaking the Waves in Science Studies: Comment on H.M. Collins and Robert Evans, 'The Third Wave of Science Studies ", Social Studies of Science, vol. 33 / 3, 2003, p. 389-400. PESTRE D., " Des sciences, des techniques et de l'ordre démocratique et participatif », Participations, vol. 1 / 1, 2011, pp. 210-238.

${ }^{17}$ BERARD Y. et ROGER A., " Ronds-points théoriques et passages à niveau analytiques. La sociologie politique peut-elle rencontrer la sociologie des sciences ? ", Politix 111/3, 2015, p. 9-26. CHALAS Y., GILBERT C., VINCK D., Comment les acteurs s'arrangent avec l'incertitude, Archives contemporaines, 2009. FRICKEL S., MOORE K., The new political sociology of science, University of Wisconsin Press, 2006. BENAMOUZIG D., BESANCON J., " Administrer un monde incertain : les nouvelles bureaucraties techniques : Le cas des agences sanitaires en France ", Sociologie du Travail, 2005, 47 (3), pp.301-322.
} 
les mandatent ${ }^{18}$. En filigrane, l'étude des controverses montre la politisation des savoirs produits, mais aussi le maintien de zones $\mathrm{d}^{\prime}$ ignorance ${ }^{19}$ et le travail d'édification de frontières ${ }^{20}$ opéré par certains acteurs au sein d'une discipline et entre les disciples scientifiques pour délimiter l'objet de la controverse. Ainsi, en analysant l'incertitude dans l'action publique, l'article souligne le processus de sélection et de légitimation de l'expertise et sa relation avec la participation publique ${ }^{21}$. II critique la thèse de la " troisième vague $~^{22}$ qui prône une limitation de la participation publique à l'extérieur du forum scientifique, tout d'abord parce qu'elle laisse de côté la signification publique d'une controverse et ensuite parce qu'elle donne aux seuls experts sélectionnés le pouvoir d'attribuer un sens politique à la controverse. ${ }^{23}$

Le choix de la comparaison

Le choix de cas français et québécois a été guidé par la proximité linguistique et la continuité temporelle de la controverse sur ces deux terrains - le sujet émerge au Québec en 2010 et en France début 2011 - qui facilitent les transferts et optimisent la comparaison des savoirs mobilisés. La trajectoire de la controverse sur ces terrains est ensuite intéressante puisqu'alors qu'en France la polémique accède directement à l'agenda politique et médiatique, le même processus prend plus de temps au Québec. Plus encore, la position des élus québécois est plus ambigüe puisque malgré des informations critiques à disposition, ils maintiennent un relatif support à l'industrie alors qu'en France les élus sont agnostiques ou critiques de l'industrie malgré des rapports plutôt partisans de la filière. De même, l'action publique engagée au regard de l'incertitude est radicalement opposée puisque Paris vote l'interdiction de la fracturation hydraulique alors que Québec encadre son utilisation et supporte l'exploration des hydrocarbures. Ensuite, en France, malgré les tentatives de différentes missions d'information, la controverse est finalement peu informée scientifiquement. En revanche, au Québec, le processus de débat public et d'évaluation environnementale dure quatre ans et donne lieu à des centaines de pages d'expertise contradictoire. Ainsi, alors que l'incertitude semble bien réduite au Québec, on ne peut pas en dire de même pour le cas français, ce qui permet d'interroger les structures institutionnelles de gestion de l'incertitude sur ces deux terrains.

\footnotetext{
${ }^{18}$ RESTIER-MELLERAY C., "Experts et expertise scientifique. Le cas de la France », Revue française de science politique, vol. 40/4, 1990, pp. 546-585.

${ }^{19}$ GROSS M., MCGOEY L., Routledge international handbook of ignorance studies, Routledge, 2015; HESS D., Undone science, MIT Press, 2016; JOUZEL J.-N., Des toxiques invisibles, Paris, EHESS, 2013.

${ }^{20}$ GIERYN T., "Boundary-Work and the Demarcation of Science from Non-Science: Strains and Interests in Professional Ideologies of Scientists", American Sociological Review, vol. 48/6, 1983, pp. 781-795.

${ }^{21}$ Suivant la distinction d'Habermas, la participation publique comprend ici les dispositifs officiels de participation du public à la décision (élections, référendums, audiences publiques) et les outils informels de débat (réunions publiques, manifestations).

${ }^{22}$ COLLINS H. M., EVANS R., "The Third Wave of Science Studies Studies of Expertise and Experience ", Social Studies of Science, vol. 32 / 2, 2002, pp. 235-296.

${ }^{23}$ Sur ce débat structurant la sociologie des sciences, voir le numéro spécial de Social Studies of Science, vol. 33/3, 2003.
} 
Enfin, malgré les différences institutionnelles - dans un cas un Etat dans l'autre une province - les deux terrains sont pleinement comparables ${ }^{24}$ puisque le Québec dispose des mêmes compétences que la France en matière de gestion de l'environnement et des ressources naturelles (avec un droit du sous-sol similaire), le niveau fédéral n'intervenant pas durant la controverse, si ce n'est de la même manière que l'Union Européenne pour la France, à travers la disponibilité de certaines expertises et la comparaison avec d'autres États membres. Cette particularité permet de réduire les difficultés d'une comparaison multiniveau ${ }^{25}$, puisque les cas étudiés permettent de centrer l'analyse sur le même niveau de gouvernement pour expliquer la construction de l'action publique.

\section{Méthodologie}

II s'agit d'étudier la controverse entre 2010 et 2015 et de comprendre quels sont les mécanismes mis en œuvre pour tenter de réduire l'incertitude, quel type d'incertitude est ciblé, par quels outils, par quels acteurs et avec quels effets sur la trajectoire politique de la controverse. L'analyse s'appuie sur trois rapports québécois (deux du Bureau d'Audiences Publiques sur l'Environnement (BAPE) et un rapport de synthèse de l'Évaluation Environnementale Stratégique (ÉES)) et quatre rapports français (un rapport d'expertise administrative du Conseil Général de I'Industrie, de l'Énergie et des Technologies (CGIET) et du Conseil Général de l'Environnement et du Développement Durable (CGEDD); un rapport d'information parlementaire (Gonnot-Martin), un rapport sur la proposition de loi (Havard-Chanteguet) ; et le rapport de l'Office Parlementaire d'Évaluation des Choix Scientifiques et Technologiques (OPECST) ${ }^{26}$ ). Une analyse qualitative de ces rapports démontre quel traitement est réservé aux discours concurrents par les commissions d'enquête et sur quelles justifications elles appuient leurs conclusions. Une analyse quantitative des 1100 entretiens et des 1385 références bibliographiques des rapports permet de faire

\footnotetext{
${ }^{24}$ HASSENTEUFEL P., « Deux ou trois choses que je sais d'elle. Quelques enseignements tirés d'expériences de comparaison européenne », in CURAPP (ed.), Les méthodes au concret, Paris, Presses universitaires de France, 2000, p. 105-124

${ }^{25}$ BOUSSAGUET L., DUPUY C., « L'analyse des politiques publiques à l'épreuve de la comparaison », Revue internationale de politique comparée, vol. 21/2, 2014, pp. 97-119.

${ }^{26}$ BUREAU D'AUDIENCES PUBLIQUES SUR L'ENVIRONNEMENT, « Le développement durable de l'industrie des gaz de schiste au Québec », Gouvernement du Québec, 2011. BUREAU D'AUDIENCES PUBLIQUES SUR L'ENVIRONNEMENT, " Les enjeux liés à l'exploration et l'exploitation du gaz de schiste dans le shale d'Utica des basses-terres du SaintLaurent ", Québec, Gouvernement du Québec, 2014. COMITÉ DE L'ÉVALUATION ENVIRONNEMENTALE STRATÉGIQUE, «Evaluation environnementale stratégique sur le gaz de schiste », Gouvernement du Québec, 2014. CONSEIL GÉNÉRAL DE L'INDUSTRIE, DE L'ENERGIE ET DES TECHNOLOGIES, CONSEIL GÉNÉRAL DE L'ENVIRONNEMENT ET DU DÉVELOPPEMENT DURABLE, "Les hydrocarbures de roche-mère en France », République française, 2012. GONNOT F.M., MARTIN P., " Les gaz et huile de schiste », Paris, Commission du développement durable et de l'aménagement du territoire, 2011. CHANTEGUET J.-P., HAVARD M., « La proposition de loi, visant à interdire l'exploration et l'exploitation des mines d'hydrocarbures liquides ou gazeux par fracturation hydraulique et à abroger les permis exclusifs de recherches comportant des projets ayant recours à cette technique ", Paris, Commission du développement durable et de l'aménagement du territoire, 2011. OFFICE PARLEMENTAIRE D'ÉVALUATION DES CHOIX SCIENTIFIQUES ET TECHNOLOGIQUES, « Les techniques alternatives à la fracturation hydraulique pour l'exploration et l'exploitation des hydrocarbures non conventionnels », Paris, Assemblée nationale et Sénat, 2013.
} 
émerger les catégories d'acteurs et de savoirs mobilisés dans la controverse. Les individus auditionnés et les sources bibliographiques sont classés en fonction de leur appartenance à l'administration, à l'industrie, aux associations environnementales ou à des instituts de recherche afin de souligner le poids relatif de chaque catégorie d'acteurs. L'analyse s'appuie également sur 40 entretiens réalisés entre 2012 et 2014 auprès de différents acteurs de la controverse, ainsi que sur divers documents produits par les parties prenantes.

L'article présente d'abord les différentes formes de savoirs et d'expertise mobilisés durant la controverse par les acteurs qui y prennent part. II souligne ensuite le processus de construction sociale de l'incertitude utilisée pour renforcer ou réduire les risques liés à l'industrie. Puis, les rapports officiels et les discours sont analysés afin d'une part de comparer les cadrages nationaux de la controverse et d'autre part de montrer les différentes manières dont les expertises ont été produites et utilisées. Enfin, l'article souligne l'aspect politique - plutôt que scientifique - de l'incertitude dans la gestion de l'action publique puisqu'il ne s'agit pas de combler une lacune objective des connaissances mais de justifier une solution politique.

\section{La construction sociale de la certitude et de l'incertitude}

La France et le Québec n'ont pas bénéficié du même " miracle » du gaz de schiste que les États-Unis. Les deux territoires sont pourtant crédités par le département américain de l'énergie de ressources importantes mais ce potentiel n'a jamais pu être exploité. En effet, des mobilisations sociales de grande ampleur ont empêché le développement de l'industrie du gaz de schiste. En France, une mobilisation fulgurante ${ }^{27}$ parvient à enrôler les élus locaux et nationaux afin de bannir la technique contestée de la fracturation hydraulique dès 2011 sans prendre le temps de l'évaluation. Il devient ensuite très difficile pour les partisans de la filière de rouvrir le débat afin d'obtenir des autorisations d'expérimentations et d'exploration de la ressource. Au Québec, la croissance de la mobilisation et d'une expertise alternative pousse le gouvernement à accepter la tenue d'audiences publiques en 2010, puis celle d'une évaluation scientifique entre 2011 et $2014^{28}$. Malgré un début d'exploration réussie, l'industrie doit arrêter ses travaux ; elle est par la suite incapable de se relancer puisque l'évaluation environnementale l'a décrédibilisée, et parce que le prix du gaz a chuté drastiquement entre 2008 et 2012. Dans ce cadre, il importe de décrire minutieusement les récits en concurrence dans les deux cas, ainsi que le type d'expertise les soutient.

\section{Encadré 1 : évènements principaux de la controverse}

\begin{tabular}{|l|l}
\hline Québec & France
\end{tabular}

\footnotetext{
${ }^{27}$ CHATEAURAYNAUD F., DEBAZ J., « L'affaire des gaz de schiste. Anatomie d'une mobilisation fulgurante ", 2011 [en ligne: https://socioargu.hypotheses.org/3262 - consulté le 30/09/16].

${ }^{28}$ CHAILLEUX S., " Construction de la controverse sociotechnique sur le gaz de schiste au Québec ", Recherches sociographiques, vol. 56/2-3, 2015, pp.325-351.
} 


\begin{tabular}{|l|l|}
\hline $\begin{array}{l}\text { 09/2009: premières revendications pour un } \\
\text { moratoire }\end{array}$ & $12 / 2010:$ premières réunions publiques \\
\hline 09/2010-02/2011 : enquête du BAPE & $02 / 2011$ : mission d'information administrative \\
\hline $\begin{array}{l}\text { 2011-2014: évaluation environnementale } \\
\text { stratégique }\end{array}$ & $03 / 2011:$ mission d'information parlementaire \\
\hline $\begin{array}{l}\text { 07/2014: réforme du Règlement sur le } \\
\text { prélèvement des eaux et leur protection }\end{array}$ & $\begin{array}{l}04 / 2011: \text { examen de la proposition de loi } \\
\text { interdisant la fracturation hydraulique }\end{array}$ \\
\hline $\begin{array}{l}\text { 06-11/2014 : seconde enquête du BAPE la Loi sur les } \\
\text { 12/2016: adoption de la } \\
\text { hydrocarbures }\end{array}$ & $\begin{array}{l}07 / 2011: 2013: \text { rapport de l'OPECST } \\
\text { hydraulique et abrogation des permis }\end{array}$ \\
\hline
\end{tabular}

L'exploration du gaz de schiste : forces et faiblesses de la certitude géoscientifique

Le développement de l'industrie est avant tout fondé sur et cadré par l'expertise de la géologie et de l'ingénierie qui produisent les connaissances relatives aux ressources potentielles du sous-sol et aux techniques adéquates pour les extraire. Le gaz de schiste devient un hydrocarbure rentable à exploiter à partir de la hausse importante des prix de l'énergie durant les années 2000 . Certaines petites compagnies ont amélioré et combiné des techniques de forage - la fracturation hydraulique et le forage horizontal - pour extraire cet hydrocarbure de roche-mère (auparavant trop coûteux à exploiter). Mais à partir de 2005-2006, les grandes compagnies s'intéressent aussi à cette ressource et cherchent à obtenir des actifs dans les pays où il leur semble le plus intéressant d'exploiter des gisements. En raison du développement précoce de cette industrie aux États-Unis et au Canada, une grande partie du savoir-faire provient de l'Alberta et du Texas. Les techniques d'exploitation ont été développées aux États-Unis - la fracturation hydraulique a été améliorée par Halliburton bien que Schlumberger ait été une société pionnière en matière de forage horizontal. En France, les permis de gaz de schiste ont été accordés à des partenariats franco-américains : Total s'est joint à Devon Energy tandis que Schuepbach Energy s'est associé à GDF-Suez (aujourd'hui ENGIE). Au Québec, de nombreuses compagnies viennent d'Alberta mais d'autres sont créées par des ingénieurs québécois issus de l'ancienne société d'État qui détenait les permis d'exploration. Alors que seulement trois permis visent spécifiquement le gaz de schiste en France dans le Sud-Est (plus une douzaine de permis dans le bassin parisien pour du pétrole de schiste), le BAPE parle de plus d'une centaine de permis au Québec entre Montréal et Québec accordés à 26 compagnies différentes à l'été 2010. 
L'industrie naissante sur les deux terrains est composée d'un mélange de petites sociétés pionnières (les juniors) et de grandes compagnies (les majors) qui tentent d'importer les techniques de fracturation hydraulique et de forage horizontal afin d'explorer de nouvelles couches du sous-sol et de s'implanter sur de nouveaux territoires. Les juniors, comme Schuepbach, ont tendance à chercher une validation rapide des ressources pour revendre les permis à des sociétés d'exploitation tandis que les majors, comme Total, cherchent à rattraper le retard dans la maîtrise technologique dû à leur arrivée relativement tardive (après 2006) dans le secteur. Pour ces compagnies, le terme «non conventionnel » appliqué à la ressource n'est pas un enjeu à cette époque : la fracturation hydraulique n'est pas nouvelle puisqu'elle est utilisée pour réactiver des puits conventionnels depuis longtemps; la phase exploratoire n'est pas considérée comme problématique puisqu'elles cherchent à valider un potentiel et que le taux de réussite est faible; les pratiques de l'industrie sont perçues comme éprouvées depuis les années 1950. Elles insistent sur le fait qu'elles recherchent du gaz naturel, mais aucunement sur les spécificités du gaz de schiste. Le cadrage par la géologie et la définition mouvante au cours du temps et de l'espace ${ }^{29}$ voilent ainsi dans un premier temps le potentiel de controverse.

Durant une phase antérieure à la controverse publique, ces sociétés doivent ajuster les techniques utilisées aux États-Unis au sous-sol local et cartographier les ressources potentielles qui ont seulement été supposées d'après l'expérience américaine avec des sous-sols similaires. La communication des entreprises est alors principalement orientée vers leurs investisseurs : il est crucial de les rassurer en prouvant la présence de gaz et en démontrant leur capacité à l'extraire. Les compagnies gazières mettent alors les géologues au travail pour cartographier le sous-sol, récupérer les données existantes auprès de l'administration, produire des représentations $3 \mathrm{D}$ des couches de roche-mère et montrer la présence de gaz dans des zones spécifiques avec l'aide de camions sismiques. A partir de ces informations, les compagnies peuvent produire des estimations économiques sur la rentabilité des forages. Au Québec, les premiers forages exploratoires confirment le potentiel gazier en 2008 lorsque le prix du gaz est à son niveau le plus haut (soit 13US\$/MBTU (Million of British Termal Unit)). Plusieurs compagnies prouvent ainsi à la fois la présence de gaz et leur capacité à l'extraire et s'activent pour développer leur financement. ${ }^{30}$ Le Québec se trouve donc dans une phase d'exploration avancée lorsque les médias généralistes s'intéressent à ce sujet. Par contraste, dans le cas français, l'étendue des ressources en gaz de schiste et la capacité technique à les exploiter demeurent incertaines puisque les compagnies n'en sont qu'à une phase précoce d'exploration (principalement à partir des données existantes) lorsque l'interdiction de la fracturation hydraulique vient stopper leur activité. Aucun forage n'a pu avoir lieu et aucune estimation crédible n'a pu être produite avant que les permis soient abrogés en 2011. Le Bureau de l'Exploration-Production Hydrocarbures (BEPH) du ministère de l'Écologie et

${ }^{29}$ L'offshore profond était considéré non conventionnel dans les années 1990, il est conventionnel aujourd'hui.

${ }^{30}$ Entretien avec un membre de l'Association des Pétrolières et Gazières du Québec, avril 2013 
les compagnies affichent leur confiance dans le potentiel mais aucune donnée directe sur le gaz de schiste n'est produite.

Cette différence en matière d'incertitude géologique a influencé l'action publique dans deux directions opposées. Au Québec, les compagnies ont prouvé que la ressource était présente et qu'elles pouvaient l'exploiter. 29 puits ont été forés et la moitié a été fracturée hydrauliquement. Pour les décideurs politiques, le gaz de schiste est tangible, ce qui facilite le soutien déjà apporté par le gouvernement Libéral de Jean Charest $^{31}$. L'exemple du voisin américain motive le gouvernement de Québec qui espère profiter de cette manne que la ministre des Ressources naturelles et de la Faune (MRNF), Nathalie Normandeau, qualifie en $2010 d^{\prime}$ « opportunité à ne pas manquer » ${ }^{32}$. Les données produites montrent en effet que le Québec pourrait avoir son propre miracle gazier. Le MRNF parle de 10000 emplois potentiels, de la hausse des redevances et taxes et d'indépendance énergétique ${ }^{33}$. Le gouvernement focalise alors ses discours sur les bénéfices économiques de l'industrie et non sur ses risques. Plus encore, le MNRF réduit les risques liés à la fracturation hydraulique dans son document de 2010 : la quantité d'eau nécessaire est jugée supportable par les rivières concernées, le document parle d'additifs et non de produits chimiques pour le fluide de fracturation, et aucun des risques soulignés par les opposants n'est mentionné. L'incertitude principale concerne les conséquences économiques que le gouvernement et les compagnies, aidés par des firmes de consultants qui produisent des prospectives économiques pour la province, s'efforcent d'afficher comme une opportunité inédite ${ }^{34}$.

En France, l'industrie est arrêtée durant la phase d'exploration précoce. Aucune donnée géologique nouvelle sur les couches de schiste n'a été produite et la présence de gaz de schiste reste spéculative. En raison de ce manque de données géologiques, aucune prévision économique ne peut être produite. Les données économiques produites en 2012 par Sia Partners ne sont en réalité que déduites de l'expérience américaine sur la base des mêmes caractéristiques du sous-sol ${ }^{35}$. De plus, comme les compagnies n'ont pas été capables de forer des puits d'exploration, elles n'ont pas été en mesure de démontrer leur capacité technique et leur usage sécuritaire de la fracturation hydraulique dans le cadre de l'exploration de gaz de schiste $^{36}$ avant le

\footnotetext{
${ }^{31}$ C'est ce gouvernement qui a dissout la société d'État auparavant détentrice d'un monopole sur les permis d'exploration d'hydrocarbures et a permis leur octroi à de nombreuses sociétés privées entre 2004 et 2006.

32 SHIELD, A., « Gaz de schiste : Normandeau fait vibrer des cordes sensibles », Le Devoir, 15 septembre 2010.

${ }^{33}$ MINISTÈRE DES RESSOURCES NATURELLES ET DE LA FAUNE, " Le développement du gaz de schiste au Québec ", Gouvernement du Québec, 2010.

${ }^{34}$ La firme Secor va même jusqu'à présenter un scénario de création de 200000 emplois sur 14 ans. SECOR, "Évaluation des retombées économiques du développement des shales de l'Utica », 2010. MACKIE RESEARCH CAPITAL CORPORATION, Spotlight on the Utica Shale: Next Steps - The Path to Commercialization, 2010.

${ }^{35}$ SIA PARTNERS, « Les Gaz Non Conventionnels : un potentiel d'emplois évalué à 100000 en France d'ici 2020 », 2012.

${ }^{36}$ Pour autant, la fracturation hydraulique a déjà été utilisée en France pour des forages sur des gisements conventionnels pour réactiver des puits. Elle est une technique classique lorsqu'elle est utilisée sur un forage vertical pour l'administration et pour les compagnies.
} 
vote de l'interdiction de 2011. Ces mêmes compagnies ont aussi parfois un discours contradictoire puisque certaines mobilisent le succès américain comme preuve de l'opportunité industrielle. C'est le cas de Schuepbach qui montre les photos de forages gaziers en plein centre-ville aux États-Unis. Mais dans le même temps, la ministre de l'Écologie, N. Kosciusko-Morizet, affirme « qu'il n'est pas question d'exploiter les gaz de schiste comme cela se fait aux États-Unis ${ }^{37}$. L'association de l'exploration française à l'expérience américaine, le manque de preuves tangibles sur les ressources et le désintérêt d'une majorité d'élus nationaux conduisent à un manque de réaction politique en faveur de cette industrie. Le potentiel à défendre n'existe pas et les hydrocarbures ne sont aucunement perçus comme dignes d'intérêt politique à cette époque. En effet, peu d'acteurs politiques militent pour cette industrie perçue comme marginale et déclinante. A rebours du Québec où l'association des pétrolières et gazières (APGQ) est créée en 2009 et se place en première ligne pour la défense de l'industrie, en France, l'Union française de l'industrie pétrolière (UFIP) est particulièrement silencieuse durant les premiers mois de la controverse puisque la branche exploration est marginale dans ce syndicat dominé par les raffineurs et les exploitants. A l'inverse du gouvernement québécois, l'incertitude sur les effets économiques et environnementaux et la dépolitisation initiale du sujet empêchent tout soutien politique à un an de l'élection présidentielle de 2012, et l'objectif des opposants va être de maintenir cette incertitude.

Enfin, il est intéressant de noter qu'à ce stade le secteur des hydrocarbures est, sur les deux terrains, organisé autour d'une législation relativement favorable aux industriels. La Loi sur les mines du Québec a préséance sur les autres législations, notamment municipales et environnementales, tandis que le Code minier français institutionnalise une délivrance des permis autour d'un dialogue entre compagnies exploitantes et administration sans consultation d'autres acteurs lors de la phase exploratoire ${ }^{38}$. Ce cadrage restreint autour d'un réseau d'acteurs relativement fermé oriente l'expertise légitime principalement vers la géologie et l'ingénierie - affirmé en France par l'évaluation de la "capacité financière et technique » des détenteurs de permis par le BEPH. Ce secteur d'action publique exprime ainsi une gouvernance restreinte définie par P. Culpepper comme "quiet politics » ${ }^{39}$ puisqu'il s'agit jusqu'alors d'une industrie relativement marginale et discrète qui suit les procédures administratives mises en place précisément pour éviter une mise en problème de ces activités. Ce sont majoritairement les acteurs économiques qui influencent la législation en vigueur ainsi que les connaissances scientifiques valorisées pour évaluer les projets. Sur les deux terrains, la controverse met en lumière l'absence de recherche sur les effets de cette industrie,

\footnotetext{
${ }^{37}$ KOSCIUSKO-MORIZET N., Journal de l'Assemblée Nationale, 2 février 2011

38 Il faut néanmoins noter l'existence de procédures plus inclusives lors de la phase d'exploitation et d'évaluation des risques lors de la demande d'autorisation des travaux, mais qui arrivent en aval du développement des projets.

${ }^{39}$ CULPEPPER P., Quiet politics and business power: corporate control in Europe and Japan, Cambridge, Cambridge University Press, 2010
} 
notamment au regard de l'hydrogéologie, illustrant une production d'ignorance ${ }^{40}$ exemplaire d'un sujet peu politisé. Le cadre institutionnel est ainsi questionné mais sa réforme s'avère complexe malgré le passage d'un espace de décision discret vers un espace public. Une différence de taille doit cependant être mentionnée : en France, c'est le ministère de l'Ecologie qui délivre les permis depuis 2007 avec une focale environnementale de plus en plus affirmée, tandis qu'au Québec c'est un ministère dédié qui octroie les permis et s'affiche ostensiblement en supporteur des activités d'extraction et d'exploration.

\section{Encadré 2 : éléments de politique québécoise}

Le gouvernement libéral de J. Charest (2003-2012) est mis en difficulté sur le gaz de schiste à partir de 2010 puisqu'il fait montre d'une faible maîtrise du dossier, de son incapacité à gérer des problèmes de fuite de méthane sur les puits forés et surtout d'une connivence à l'égard de l'industrie qui débauche de nombreux libéraux comme lobbyistes. II chute par la suite en 2012 après les manifestations dites du « Printemps Erable " causées par l'augmentation programmée des frais de scolarité. Un gouvernement minoritaire du Parti Québécois est alors élu avec à sa tête P. Marois qui s'affiche hostile au gaz de schiste, mais favorable au pétrole de schiste dont il négocie un partenariat d'exploration sur l'île d'Anticosti avec des sociétés pétrolières. Défait à son tour en avril 2014, ce gouvernement est remplacé par celui du libéral P. Couillard dont les propos sur les hydrocarbures sont contradictoires, tantôt en faveur des projets d'exploration, tantôt les critiquant comme lors de la COP21. II fait cependant voter la Loi sur les hydrocarbures en 2016 qui vient réaffirmer ce que les orientations énergétiques de 2015 avaient annoncé c'est-à-dire l'ouverture de la province aux projets d'hydrocarbures afin de diversifier le mix énergétique tout en mettant en place un cadre réglementaire strict.

\section{Croissance de la mobilisation et ouverture de l'expertise}

La controverse s'étend lentement au Québec mais rapidement en France alors que la certitude des géosciences est attaquée par d'autres types d'incertitudes relatives à la santé publique, aux effets environnementaux ou au cadre légal. En France, le mouvement d'opposition est initié en décembre 2010 par des journalistes et des élus écologistes et socialistes du Sud-Est. Au député européen José Bové, viennent s'ajouter les socialistes Michèle Eybalin du Conseil régional de Rhône-Alpes, ou Pascal Terrasse, député et président du Conseil départemental de l'Ardèche, ainsi qu'un collectif de maires ardéchois. Mais les quelques élus et militants impliqués initialement sont rapidement dépassés par la création de plus de 180 collectifs citoyens dans les premiers mois de $2011^{41}$. Chaque collectif organise des réunions publiques, diffuse le documentaire Gasland (réalisé par l'Américain Josh Fox qui montre les ravages de l'industrie aux

\footnotetext{
40 HESS, op. cit.

${ }^{41}$ SEGUIN L., Les apprentissages de la participation. Regards croisés sur un dispositif institué et une mobilisation contestataire. Thèse pour le doctorat de sociologie. Université François Rabelais - Tours, 2016.
} 
Etats-Unis) et participe à des manifestations. Le risque pour l'eau est le fer de lance des revendications, mais ces opposants dénoncent aussi l'opacité du processus administratif qui n'a pas permis d'organiser des consultations préalables, et plus timidement la lutte contre les changements climatiques. Les opposants français ne réclament pas de moratoire mais une interdiction pure et simple de l'industrie et l'annulation des permis. Le gouvernement réagit promptement dès le 2 février 2011 alors que la première réunion publique a lieu le 20 décembre 2010, et en avril débute un processus législatif qui scelle le sort du gaz de schiste. Au Québec, l'alerte percole au contraire lentement entre la demande de moratoire de l'Association Québécoise de Lutte contre la Pollution Atmosphérique en septembre 2009 et le déclenchement des audiences du BAPE en septembre 2010. Les opposants construisent un récit qui cadre l'industrie du gaz de schiste comme une menace pour l'environnement et la santé publique et ils dénoncent la précipitation, le cadre légal inadéquat et le manque de consultation. Ils revendiquent un moratoire pour évaluer les risques et les opportunités.

Sur les deux terrains, les opposants au gaz de schiste réussissent à émettre une alerte dans le forum médiatique et politique en utilisant l'incertitude comme une ressource politique. Ils parviennent à rendre public un sujet souterrain et la lumière projetée sur le sous-sol questionne les types d'expertise mobilisés par l'administration pour évaluer ces activités d'extraction. Deux stratégies peuvent être mises en avant: rendre incertaines les données géologiques et techniques et accentuer les risques en ouvrant la controverse à de nouveaux domaines comme la pollution des nappes phréatiques, la santé publique, la gouvernance ou l'acceptabilité sociale.

D'abord, l'expertise mise en avant jusque-là par les compagnies et l'administration est accusée d'être partiale: "On a compris que l'information à laquelle le gouvernement avait accès était totalement insuffisante. J'ai demandé aux représentants des ministères : d'où vient l'information ? Et ils m'ont répondu : nous l'avons des compagnies. » ${ }^{42}$ Pour contredire ces informations, sur les deux terrains, les opposants enrôlent des experts géologues et ingénieurs, souvent retraités de l'industrie ou de centres de recherche. Ce sont des experts légitimes sur le sujet mais ils sont pourtant exclus par les experts professionnels qui les accusent de ne pas connaître ce sujet spécifique ou de ne plus être à jour sur les technologies. Le parcours de deux experts critiques met en avant les dynamiques qui soutiennent leurs actions. Un géologue de l'Université du Québec à Montréal devient une figure importante du mouvement d'opposition québécois ; à ce titre, il subit des campagnes de dénigrement par l'industrie qui l'accuse de ne pas être un géologue pétrolier. II gagne toutefois en crédibilité dans les médias et parmi les opposants, et il parvient même à rencontrer la ministre des Ressources naturelles après le changement de gouvernement de l'automne 2012. II participe à de nombreuses interviews et réunions publiques dans lesquelles il critique les données géologiques, souligne les risques liés à la fracturation hydraulique, et met en doute les données

\footnotetext{
${ }^{42}$ Entretien avec un membre du collectif scientifique sur le gaz de schiste, juin 2012
} 
économiques. Sa voix porte notamment sur les réflexions de la commission sur l'avenir énergétique de la province en 2013, mais il ne parvient pas à convaincre les ministres libéraux. En France, dès janvier 2011, un groupe de géologues et d'hydrogéologues de l'Université de Montpellier publie le premier document scientifique sur le gaz de schiste en France ${ }^{43}$. Si un géologue émet des doutes sur la présence de gaz rentable dans le Sud-Est, un des hydrogéologues souligne plutôt le manque de considération accordée à la nature karstique du sous-sol dans ces zones qui rend difficile la cimentation des puits. II participe par la suite à diverses conférences ; il influence également les rapports officiels pour lesquels il a été auditionné et qui réclament des études plus poussées sur la topographie du sous-sol du Sud-Est ${ }^{44}$. Son travail participe à contrecarrer la production de la certitude géologique faiblement défendu en France durant les premiers mois de 2011. Malgré la confiance du BEPH dans les techniques et la présence de ressources, les décideurs politiques font désormais face à des interprétations divergentes des données existantes et à un besoin de connaissances nouvelles.

Dans le même temps, d'autres experts visent à étendre le périmètre d'enquête à leur propre discipline scientifique et professionnelle. Au Québec, où cette diversification est plus marquée, un collectif scientifique alternatif est créé en janvier 2011 ; il regroupe plus de 170 professeurs, rassemblant des spécialistes du Droit, de l'Economie ou de la Santé publique. Certains produisent des outils pour les opposants tel que le Règlement Saint-Bonaventure, un arrêté municipal rédigé en 2011 par un juriste qui profite d'une faille dans la règlementation sur l'eau pour empêcher les compagnies d'introduire des produits chimiques dans le sol à proximité des forages d'eau potable. Plus d'une centaine de règlements similaires sont alors adoptés dans son sillage et focalisent la controverse sur le droit des municipalités, en enrôlant les élus locaux dans la lutte. ${ }^{45}$ Des économistes universitaires démystifient les données produites par les firmes de consultants. Un toxicologue français souligne dès mai 2011 les dangers relatifs aux produits chimiques utilisés pour la fracturation hydraulique et l'Institut national de la santé publique du Québec insiste en 2013 sur les risques pour la santé publique posés par l'industrie, participant à valider les craintes des riverains. Bien que certaines expertises aient été contredites par la suite, ces disciplines alternatives ouvrent le débat et la controverse n'est plus alors seulement une question de données géologiques et de maîtrise technologique. En France, le cadrage demeure longtemps focalisé sur les géosciences et les questions de l'eau jusqu'en 2012-2013 où apparaissent alors des préoccupations plus économiques ${ }^{46}$. Les autres aspects, relatifs à la gouvernance notamment, sont difficilement politisés par les opposants qui sont à la fois démobilisés dans un

\footnotetext{
${ }^{43}$ MOLINATTI G., SIMONNEAU L., op. cit.

${ }^{44}$ CONSEIL GÉNÉRAL DE L'INDUSTRIE, DE L'ENERGIE ET DES TECHNOLOGIES, CONSEIL GÉNÉRAL DE L'ENVIRONNEMENT ET DU DÉVELOPPEMENT DURABLE, op. cit.

${ }^{45}$ Une partie des revendications des municipalités intègre les débats sur la réforme de la Loi sur les mines en 2013 (possibilité de retirer des zones à la prospection) avant d'être contrecarrée par la réforme du Règlement sur le prélèvement des eaux et leur protection en 2014 (interdiction de réglementer le secteur des hydrocarbures).

${ }^{46}$ PORCHER T., Le mirage du gaz de schiste, Paris, Max Milo, 2013
} 
premier temps par la Loi Jacob de 2011 qui interdit la fracturation hydraulique, puis désorganisés autour des questions annexes sur la réforme du Code minier et la promotion de la transition énergétique (avec une scission entre des groupes uniquement intéressés par la fracturation et ses dangers et d'autres qui développent une lutte globale contre les hydrocarbures) ${ }^{47}$. C'est bien la visibilité médiatique et la cohérence avec les préoccupations des acteurs qui permettent, à un moment de la controverse, de valoriser ces expertises alternatives jusque-là plutôt discrètes.

Face aux attaques portant sur la légitimité de leur expertise, les industriels rejettent généralement celle mobilisée par les opposants, arguant d'un manque de connaissance et de biais idéologiques. "Nous estimons qu'il est nécessaire d'avoir un diplôme académique et 20 ans d'expérience pour comprendre [les enjeux du gaz de schiste], maintenant on a à faire à des gens qui pensent qu'ils peuvent tout comprendre après dix minutes sur internet. ${ }^{48} C^{\prime}$ est encore plus remarquable en France où apparaît une opposition manichéenne entre l'obscurantisme et le progrès de la connaissance à la suite du vote de l'interdiction de la fracturation hydraulique en juillet 2011. La controverse française et ses retombées sont perçues comme un déni scientifique : «Au-delà de ce feu de forêt, il nous apparaissait quand même dommage, et inhabituel je dirais par rapport à d'autres processus, de se priver d'emblée d'une possibilité d'examiner l'existence potentielle d'une ressource, de vérifier si oui ou non il y a une ressource. " ${ }^{49}$ Ce ne sont pourtant pas les compagnies gazières qui sont les plus virulentes en France à propos de la nécessité d'autoriser l'exploration, mais certains élus dont ceux de l'OPECST. Le député Bataille accuse ainsi les membres d'Europe Ecologie Les Verts d'être les «Torquemada de l'obscurantisme ». Dans le rapport de 2013, ces élus de I'OPECST dénoncent le principe de précaution et réclament des expérimentations afin de démontrer la sécurité de la fracturation hydraulique et le potentiel du sous-sol.

« On appelle gaz de schiste, le gaz méthane qui est le même que celui qu'on extrayait jusqu'à présent. Donc pour nous rien n’a changé. Les problèmes se sont révélés car ce sont les écolos qui les ont mis en exergue dès le moment où un film catastrophe est venu des États-Unis. Mais pour nous, il n'y avait pas de problème. Puisque l'extraction du gaz se faisait toujours dans les mêmes conditions, sinon que pour les gaz de schiste, l'extraction se faisait dans une roche qui n'était pas considérée par la profession comme étant susceptible d'en extraire du gaz. Dès le moment où la profession a détecté que le gaz pouvait se trouver dans cette roche, que l'on appelle roche-mère, ils ont essayé des techniques nouvelles, enfin nouvelles, qui existaient déjà, parce que la fracturation hydraulique ça a toujours existé. $)^{50}$

\footnotetext{
${ }^{47}$ Le même type de scission se produit au Québec fin 2013 mais à un stade où le mouvement a déjà établi une solide et diverse assise scientifique.

${ }^{48}$ BINNION M., The Gazette, Février 2011 (traduction de l'auteur)

${ }^{49}$ Entretien avec un membre de I'UFIP, avril 2012

${ }^{50}$ Entretien avec un membre de l'Amicale des foreurs, juin 2012
} 
En France, les partisans de la filière privilégient donc une approche technicienne de la controverse en démontrant leur maîtrise technique tandis qu'au Québec, ils visent à développer l'acceptabilité sociale des projets d'exploitation en insistant sur les bénéficies de l'industrie.

\section{Réduire ou maintenir l'incertitude : une ressource politique}

Des deux côtés de l'Atlantique, la controverse sur les hydrocarbures non conventionnels a été cadrée comme un problème d'incertitude. Cette incertitude est une ressource politique puisque sur un objet similaire, différents résultats politiques sont produits en fonction du poids des acteurs, de leur discours et des dispositifs d'action publique mis en œuvre. L'incertitude est utilisée pour orienter la controverse et sa traduction en politique publique. Alors qu'en France, le récit principal tourne autour de l'incertitude géologique et technologique, au Québec, on observe un processus de réduction des multiples sources d'incertitude ainsi qu'un confinement de l'incertitude technologique.

En France, l'incertitude est réduite par un cadrage étroit puisque la majorité des rapports officiels n'évalue que l'incertitude attachée aux ressources disponibles et aux risques de la fracturation hydraulique. Le Conseil général de l'industrie, de l'économie et des technologies (CGIET) et le Conseil général de l'environnement et du développement durable (CGEDD) affirment ainsi « qu'il serait dommageable, pour l'économie nationale et pour l'emploi, que notre pays aille jusqu'à s'interdire, sans pour autant préjuger des suites qu'il entend y donner, de disposer d'une évaluation approfondie de la richesse potentielle. ${ }^{51}$ Alors qu'une autre revendication des opposants était la réforme du Code minier et une consultation en amont des projets, le cadrage sur la fracturation hydraulique permet de dépolitiser cette réforme. Les commissions ont alors le pouvoir de rendre discutables certains aspects de la controverse ${ }^{52}$, mais aussi de rendre diffuse la décision finale $e^{53}$ en ouvrant un calendrier d'expérimentation. La mission d'expertise administrative et la mission d'information parlementaire sont toutefois mises de côté par la célérité de la réponse législative. Face à « l'incendie qui se propage ${ }^{54}$ à l'île-de-France, il faut agir vite et le chef du groupe du parlementaire UMP et député de Seine-et-Marne, Christian Jacob, dépose une proposition de loi dans I'urgence qui sera adoptée. L'interdiction de la fracturation hydraulique est ainsi imposée en juin 2011. Toutefois, la loi demeure ambiguë puisque si elle interdit la technique, elle appelle aussi de ses vœux une évaluation annuelle et des projets d'expérimentation. La loi vise donc à réduire l'incertitude soulevée par les opposants en engageant des expérimentations.

\footnotetext{
${ }^{51}$ CGIET-CGEDD, op. cit., p.73

${ }^{52}$ BARTHE Y., Le pouvoir d'indécision - la mise en politique des déchets nucléaires, Paris, Economica, 2006.

${ }^{53}$ HENRY, E., Amiante: un scandale improbable, Presses Universitaires de Rennes, 2007.

${ }^{54}$ Formulation d’un député interrogé qui a participé à la commission législative du printemps 2011.
} 
Cependant, il n'y a jusqu'à maintenant que l'interdiction qui est appliquée puisque les opposants maintiennent la pression pour empêcher toute expérimentation et réouverture du dossier. L'interdiction était pourtant initialement conçue comme une période tampon permettant de « refroidir les contestations ${ }^{55}$ et d'engager un programme de recherche rassurant - un moyen de permettre un retour dans une phase de gestion discrète. Le récit contenu dans les rapports (celui du CGIET-CGEDD ${ }^{56}$ mais aussi de I'OPECST, de Havard-Chanteguet et dans une moindre mesure de Gonnot-Martin) est celui d'une incertitude géologique et technologique qu'il faut lever par un processus d'expérimentation qui permettra ensuite de lever l'incertitude économique. Pour ces rapporteurs, c'est seulement au moment où les ressources auront été identifiées comme rentables que l'incertitude socio-environnementale pourra s'exprimer. Or, le processus politique ne se déroule aucunement de cette manière puisque l'interdiction est votée dans l'urgence. Une majorité d'élus regrettent le manque de cohérence politique et scientifique, et les rapporteurs de la mission d'information parlementaire ne manquent pas de souligner le paradoxe de voter une loi avant les résultats de leur mission. La Loi Jacob montre que les partisans n'ont pas su se mobiliser à temps pour imposer leur définition de l'industrie au public (même s'ils ont fortement imprégné les conclusions des différents rapports) et que les opposants sont parvenus à subvertir toute tentative de rouvrir le débat par la suite. Malgré la certitude technique affichée par les compagnies et leurs soutiens, celle-ci n'a pas été validée politiquement. La forte médiatisation et politisation des enjeux a rendu inopérante la gestion silencieuse de la période précédente et le poids des acteurs économiques s'est avéré insuffisant et trop incertain pour peser face à la mobilisation fulgurante soutenue par de nombreux élus locaux.

Les opposants français parviennent ainsi à empêcher la réduction de l'incertitude relative aux géosciences précisément grâce à la Loi Jacob. L'instrument de gestion de l'incertitude devient de ce fait un dispositif de production d'ignorance ${ }^{57}$. En maintenant l'incertitude sur les quantités de ressources présentes et sur la maîtrise de la fracturation hydraulique sur le territoire français, les opposants bloquent toute évaluation de la valeur économique et géostratégique de l'industrie du gaz de schiste en France. A l'inverse des vœux formulés par de nombreux rapports officiels entre 2011 et 2014, l'application partielle de la Loi Jacob supportée par les opposants a poussé le gouvernement non seulement à mettre de côté l'expérimentation relative au gaz de schiste ${ }^{58}$, mais aussi l'octroi de nouveaux permis de recherche d'hydrocarbures devenus suspects aux yeux des populations locales. Dans l'état actuel du débat, seules deux tentatives officielles ont

\footnotetext{
${ }^{55}$ BARTHE Y., LINHARDT D., « L'expérimentation: un autre agir politique ». Papiers de recherche du CSI, vol. 13, 2009.

${ }^{56}$ Il est à noter l'ambiguïté interne à ce rapport qui traduit le conflit d'interprétation de leur mission par les membres du CGIET (dépendant du ministre de l'Industrie) et du CGEDD (dépendant du ministre de l'Ecologie qui a pris la main sur le dossier).

${ }^{57}$ DEBOURDEAU, A. « Quand la solution devient le problème », Politix, 95 (3), 2011.

${ }^{58}$ F. Hollande déclarant en 2013 qu'aucune exploration n'aurait lieu durant son mandat : BAUDET, M.-B., « François Hollande et le gaz de schiste : vers un tournant majeur? ", Le Monde, 15 juillet 2013.
} 
apporté des données inédites à la controverse : celle du rapport parlementaire Barbier ${ }^{59}$ sur les effets économiques potentiels au regard de l'expérience américaine - qui s'avère assez critique sur le récit des promoteurs de l'industrie en matière de création d'emploi ou de baisse du prix du gaz; et celle du rapport Montebourg sur la fracturation au fluoropropane - le ministère du Redressement Productif ayant entrepris " en secret » l'évaluation de ce mode d'extraction qui demeure encore au stade du développement. Le cas français montre ainsi que ne pas savoir est une ressource politique tout aussi importante que savoir. Si le pouvoir d'indécision a pu être utilisé aux fins de poursuivre les projets de stockage souterrain des déchets nucléaires ${ }^{60}$, dans le cas du gaz de schiste, l'indécision bénéficie aux opposants. Les inégalités des ressources entre les industriels et les opposants sont compensées par l'importance de la mobilisation sociale et la faiblesse de l'intérêt politique envers l'industrie. Les zones d'ignorance ne sont comblées qu'indirectement: des recherches en hydrogéologie visent à cartographier des eaux souterraines tandis que les forages conventionnels permettent une relative évaluation des hydrocarbures non conventionnels grâce au carottage.

L'incertitude a été gérée de manière différente au Québec. Si l'on peut observer un travail aux frontières similaire entre les disciplines scientifiques, l'incertitude y a été réduite grâce aux travaux de l'Évaluation environnementale stratégique (ÉES), regroupant 73 études évaluant les multiples aspects du problème et disposant d'un budget de sept millions de dollars. La controverse migre ici plutôt sur le terrain des procédures administratives et politiques de nomination par le gouvernement des membres du comité d'évaluation ou encore la rédaction des questions de recherche (l'évaluation économique est mise de côté pendant un temps). Mais les opposants parviennent malgré tout à imposer certaines questions de recherche grâce à leur participation aux audiences publiques, permises par le mode de fonctionnement du BAPE. Malgré un premier cadrage restreint sur le « développement durable de l'industrie » en 2010, les opposants parviennent à pointer des nombreuses lacunes dans les connaissances. Lorsqu'il conclut à un manque de données pour produire ses recommandations, le BAPE annonce clairement que son mandat était insuffisant et demande au gouvernement de produire des données plus fiables. A ce stade, le BAPE met en doute la certitude affichée par l'industrie et le gouvernement et pointe du doigt les désaccords interministériels (entre le MRNF et le MEDDE notamment). Le gouvernement doit alors s'engager dans une évaluation environnementale stratégique permettant de réduire une incertitude plus large qui inclut l'utilisation de l'eau, la pollution souterraine et aérienne, l'activité sismique, l'acceptabilité sociale, la rentabilité économique ou le cadre légal. L'ÉES s'avère être relativement critique des arguments initiaux de maîtrise technologique et d'opportunité économique. Bien que l'incertitude sur les risques sismiques ou la

\footnotetext{
${ }^{59}$ BARBIER F., L'impact économique de l'exploitation des gaz de schiste, Paris, Commission des affaires économiques, 2014.

${ }^{60}$ BARTHE, op. cit.
} 
consommation d'eau soit réduite au profit de l'industrie, d'autres risques jusque-là ignorés apparaissent plus probables et confirment les craintes des opposants comme celui lié à l'augmentation des émissions de gaz à effet de serre en cas de développement de l'activité. En 2014, l'industrie du gaz de schiste est abandonnée à la suite des conclusions de l'ÉES et d'un second BAPE, mais aussi (et surtout) à cause de la chute du prix du gaz à $4 \$$ /MBTU. L'expertise comme ressource politique est utilisée avec succès par les opposants de la vallée du Saint-Laurent puisqu'ils ont su contredire les premières études de faisabilité, ou tout du moins retarder la mise en œuvre des projets jusqu'au retournement du marché. Ici encore, c'est par la publicisation de risques potentiels que la gestion discrète des hydrocarbures s'ouvre à des expertises alternatives.

Mais ce qui est également frappant c'est que la légitimité de l'expertise est limitée à certaines zones géographiques, en l'occurrence les basses terres du Saint-Laurent. On observe en effet une imperméabilité entre les projets relatifs aux hydrocarbures au Québec en matière de réduction de l'incertitude puisque chaque projet fait l'objet d'une évaluation ad hoc qui s'appuie peu sur les connaissances produites auparavant. Malgré les doutes émis par l'ÉES, le gouvernement Couillard a autorisé l'utilisation de la fracturation hydraulique en réformant le Règlement sur la protection des eaux et leur prélèvement en 2014, la rendant plus facile dans les territoires peu habités et plus restreinte dans ceux plus peuplés (et plus à même de se mobiliser). Ce faisant, il met fin à l'incertitude légale posée par le Règlement de SaintBonaventure en retirant aux municipalités le droit de légiférer sur les forages. Le gaz de schiste est alors abandonné principalement à cause du manque d'acceptabilité sociale dans les basses terres du SaintLaurent et à cause du bas prix du gaz. Mais dans d'autres territoires, comme à Anticosti où vivent seulement 200 personnes, la fracturation hydraulique est utilisée pour explorer le pétrole de schiste. L'ouverture du cadrage de l'incertitude a contribué à produire une évaluation globale défavorable à l'industrie mais elle n'a été appliquée qu'à une partie du territoire et le gouvernement n'a pas retenu les craintes vis-à-vis de la technologie utilisée. Le gouvernement filtre les informations légitimes et le ministère de l'Environnement tend à se référer à d'autres études américaines sur le sujet. Paradoxalement, alors qu'une plus large partie de l'incertitude relative à l'industrie du gaz de schiste a été levée au Québec en pointant de nombreux défauts, c'est une politique moins restrictive qui s'applique (la Loi sur les hydrocarbures votée en 2016, bien que relativement stricte au regard de ce qui se fait en Amérique du Nord, ouvre la province aux projets d'hydrocarbures et relance certains projets de gaz de schiste). En effet, au Québec, le ministère de l'Energie et des Ressources naturelles demeure un supporteur de l'industrie extractive qui mobilise des acteurs économiques plus importants et mieux connectés aux parlementaires.

L'incertitude peut ainsi être comprise comme une ressource politique et une production sociale. Les décideurs politiques ont d'abord fait face à une incertitude scientifique et technique : existe-t-il une ressource ? Comment peut-on l'exploiter? Mais cette incertitude est ensuite utilisée par certains acteurs 
pour faire valoir un agenda politique particulier. Les compagnies gazières minimisent l'incertitude pour attirer des investisseurs et prouver leur capacité à exploiter alors que les opposants cherchent à ouvrir des zones d'incertitude aussi bien à l'intérieur des domaines défendus par les industriels qu'à l'extérieur en cherchant à prouver des risques connexes. La force et la forme des discours ne sont pas les mêmes sur les deux terrains en fonction du poids relatifs des acteurs. Le poids économique des ressources naturelles au Québec permet de convaincre les élites politiques et économiques de l'opportunité du gaz de schiste malgré les risques potentiels. Le discours économique est favorisé par l'avancée de l'exploration qui a réduit l'incertitude géologique et technique. En France, le secteur extractif a perdu tout son poids depuis les années 1970 et l'industrie des hydrocarbures est relativement désorganisée dans sa réponse aux critiques. La contestation se produit dès les premiers balbutiements de la filière et mobilise des acteurs politiques locaux comme nationaux qui pèsent lourd dans le cadrage de la fracturation hydraulique comme un risque. Mais ces récits portés des acteurs aux ressources diverses vont surtout être passés au filtre de dispositifs institutionnels variés qui vont traduire et cadrer la controverse de manière contrastée.

\section{L'appel aux experts pour réduire quel type d'incertitude ?}

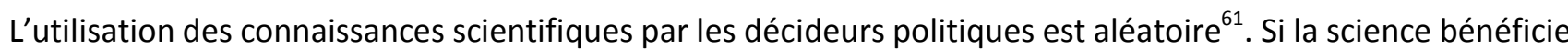
d'une supposée objectivité utilisée pour dépolitiser l'incertitude controversée ${ }^{62}$, l'appel à l'expertise et les conditions de sa légitimation montrent pourtant qu'il s'agit de pratiques politiques et d'instruments non neutres de l'action publique.

Les mandats et le cadrage institutionnel des problèmes d'incertitude

Tout d'abord, les commissions mandatées par les gouvernements cadrent chacune le problème sur une question particulière. Comme le note F. Fischer: "The process of framing is the basis for not only identifying the problem, but also for defining it. In this sense, the processes of framing predetermine the direction and nature of the technical analysis that might follow." ${ }^{63}$ En 2010, le mandat du premier BAPE au Québec focalise sur le "développement durable de l'industrie du gaz de schiste", et de plus, il vise un nombre restreint de régions administratives. Toutefois, les conclusions ouvrent les questionnements à de nouveaux problèmes. Ceux-ci sont analysés par l'ÉES à travers plus de 70 études scientifiques qui sont de nouveau portées devant le BAPE pour de secondes audiences publiques. II y a donc une ouverture dans le périmètre d'évaluation de ces trois commissions et le BAPE montre que le cadrage initial n'est pas une barrière infranchissable.

\footnotetext{
${ }^{61}$ WEISS C., Evaluation Research: Methods of Assessing Program Effectiveness, Englewood Cliffs, Prentice-Hall, 1972. WEISS C., MURPHY-GRAHAM E., PETROSINO A., [et al.], « The Fairy Godmother--and Her Warts: Making the Dream of Evidence-Based Policy Come True ", American Journal of Evaluation, vol. 29 / 1, 2008, p. 29 - 47.

62 JASANOFF S., The Fifth Branch: Science Advisers as Policymakers, Cambridge, Harvard University Press, 1990. BARTHE, Y., op. cit.; HENRY, E, op. cit.

${ }^{63}$ FISCHER F., op. cit., p. 127.
} 
Néanmoins, l'ÉES n'est pas un processus institutionnalisé au Québec et il permet au gouvernement d'influencer la composition du comité, le contenu des questions de recherche et le cadrage du mandat. Les opposants ont dénoncé le fait que sur les onze membres du comité de l'ÉES, huit étaient issus de l'administration ou de compagnies gazières. Alors que des audiences publiques sont mises en place en décembre 2011 pour discuter du plan de réalisation, les 350 personnes présentes ne parlent que de la composition biaisée du comité. Ils obtiennent finalement la démission du membre de la compagnie Junex qui est remplacé par un membre des Amis de la Terre. Les mêmes opposants critiquent aussi les orientations de l'évaluation:

"Sur les 70 études de l'EES combien viennent des ministères, combien sont pertinentes? Sur la question des risques technologiques, (...) ils passent complètement à côté de la question, ce n'est pas fortuit car dans le groupe de l'EES, il y a peu d'expertise en génie géologique. L'expert était Marianne Molgat de la compagnie Talisman. (...) J'ai demandé qui s'occupe des risques de pollution de l'eau? C'est Marianne Molgat. Qui s'occupe des risques de détérioration des puits? Marianne Molgat. Tout ce qui était des enjeux cruciaux c'était la représentante de l'industrie qui le faisait. Ils étaient onze à l'EES, sociologue, fonctionnaires du gouvernement, etc. et ils se sont fiés à la seule personne qui avait un peu d'expertise, qui était de l'industrie, qui a pu orienter le travail en évacuant certaines questions contraires à ses intérêts. " ${ }^{64}$

Les liens sont très visibles entre le forum de production scientifique et celui de la mobilisation puisque les opposants veillent à ce que l'évaluation couvre un périmètre élargi, certains opposants portent même une candidature pour participer au comité de l'EES. Dès son arrivée au pouvoir en 2012, le Parti Québécois met de côté ce comité controversé pour placer l'ÉES dans les mains du BAPE.

La première décision prise en France en février 2011 est de mandater une mission d'expertise administrative (CGIET, CGEDD) pour évaluer les ressources et engager une étude d'impact. C'est un cadre restreint d'analyse des enjeux qui n'examine que la faisabilité technique de l'industrie. De plus, la mission doit faire face aux conflits entre les quatre rapporteurs qui n'ont ni les mêmes objectifs, ni les mêmes pratiques d'expertise en fonction de leur ministre de tutelle. Une mission d'information parlementaire (GonnotMartin) suit en mars et bénéficie d'un mandat plus large. Elle analyse les pour et les contre, mais elle n'a aucun effet sur la controverse puisque son rapport n'est publié qu'après le processus législatif aboutissant à la Loi Jacob. Surtout, chaque rapporteur a sa propre conclusion diamétralement opposée. C'est la commission de travail législatif (Havard, Chanteguet) qui est la plus influente sur la politique publique française. En travaillant sur la rédaction du texte de loi, cette commission bipartite part d'un cadrage large d'une interdiction de la filière des hydrocarbures non conventionnels (version de la proposition de loi rédigée par le député Jacob) pour aboutir à un cadrage restreint d'interdiction de la seule fracturation

${ }^{64}$ Entretien avec un géologue, juillet 2014 
hydraulique. Ce choix tactique est dicté par les possibilités légales de bloquer rapidement l'industrie et " éteindre l'incendie », mais le texte de loi autorise aussi l'expérimentation, ce qui a pour effet de focaliser sur les géosciences la recherche de réponse à l'incertitude. Le dernier rapport analysé (OPECST) cadre justement son étude sur les techniques alternatives et ses conclusions sont un manifeste en faveur de l'exploration et de l'expérimentation. L'OPECST prend l'initiative de ce travail face à l'inaction du gouvernement dans la mise en place de la commission de suivi des expérimentations prévue dans la Loi Jacob mais jamais mise en œuvre. Dans le cas français, les questions posées sont « propositionnelles » : « la fracturation hydraulique est-elle sécuritaire ? " alors qu'au Québec, le questionnement porte sur le sens des projets gaziers et sur les connaissances pertinentes pour évaluer l'industrie.

La sélection des experts légitimes

Le mandat des diverses commissions a orienté les catégories d'experts appelés à fournir de l'information. Afin de montrer le type d'expertise mobilisé dans chacun des rapports, leurs références bibliographiques et les individus auditionnés sont ici analysés. Les rapports parlementaires français (Gonnot-Martin et HavardChanteguet) font principalement référence à des documents administratifs et légaux pour supporter leurs conclusions et les sources scientifiques académiques sont assez rares. Les rapports du CGIET-CGEDD et de I'OPECST sont plus intéressants de ce point de vue puisque ces enquêtes sont pensées comme devant être un état de l'art sur l'industrie du gaz de schiste ou sur les technologies employées. Leurs conclusions s'appuient en majorité sur une littérature administrative (en citant énormément des administrations étrangères comme le département américain de l'Énergie) et sur des sources scientifiques, mais également sur un large corpus de documents fournis par les compagnies gazières qui conservent un monopole d'expertise $^{65}$ sur certains aspects de la controverse (composition des fluides de fracturation, relevés sismiques, etc.). Paradoxalement, c'est le rapport de l'office parlementaire qui est le plus riche en références et en sources scientifiques académiques ( $45 \%$ de la bibliographie) devant le rapport des experts de l'administration. Mais il faut cependant prendre en considération la politique de référencement des commissions qui s'avère peu rigoureuse au dire même des rapporteurs du CGIET, illustrant la séparation entre les pratiques d'expertise académique et celle de l'administration.

Les rapports québécois bénéficient d'un recours beaucoup plus systématique à une bibliographie complète sur le sujet du gaz de schiste (entre 220 et 460 références par rapport contre moins de 100 pour les rapports français). De manière significative, le rapport du BAPE de 2014 comprend moins de citations de la littérature produite par les compagnies gazières mais aussi les associations, ce qui valide sa distanciation des

\footnotetext{
${ }^{65} \mathrm{~A}$ ce titre, le BEPH tire parti de l'exploration puisque cela lui permet de mettre à jour la cartographie du sous-sol. II fait office de bibliothèque pour les compagnies qui viennent également $y$ chercher des informations sur les forages précédents.
} 
documents partisans et la recherche d'une certaine objectivité reposant sur les travaux de l'ÉES qui le précèdent. Ce faisant, ce rapport démontre une distinction entre le forum scientifique qui produit des connaissances et le forum public qui produit de l'incertitude. On voit également une continuité dans l'action publique québécoises au regard de la cumulativité des rapports tandis que la production quasi simultanée de rapports en France est plutôt redondante.

Tableau 1 : Origine disciplinaire des sources scientifiques des rapports

\begin{tabular}{|c|c|c|c|c|c|c|c|}
\hline $\begin{array}{l}\text { Disciplines des documents } \\
\text { scientifiques cités }\end{array}$ & $\begin{array}{l}\text { BAPE } \\
2011\end{array}$ & $\begin{array}{l}\text { BAPE } \\
2014\end{array}$ & EES & GM & $\mathrm{HC}$ & $\begin{array}{l}\text { CGIET- } \\
\text { CGEDD }\end{array}$ & OPECST \\
\hline Santé publique & $1,8 \%$ & $6,2 \%$ & $1,5 \%$ & $9,1 \%$ & $0 \%$ & $6,7 \%$ & $0 \%$ \\
\hline Énergie & $2,7 \%$ & $0 \%$ & $0,7 \%$ & $9,1 \%$ & $16,7 \%$ & $13,3 \%$ & $4,9 \%$ \\
\hline Sciences sociales & $1,8 \%$ & $17,7 \%$ & $8,1 \%$ & $0 \%$ & $0 \%$ & $0 \%$ & $0 \%$ \\
\hline Droit & $2,7 \%$ & $8,8 \%$ & $6,6 \%$ & $0 \%$ & $33,3 \%$ & $0 \%$ & $0 \%$ \\
\hline Économie & $7,3 \%$ & $16,8 \%$ & $10,3 \%$ & $0 \%$ & $0 \%$ & $20 \%$ & $14,6 \%$ \\
\hline Ingénierie & $13,8 \%$ & $4,4 \%$ & $8,1 \%$ & $0 \%$ & $0 \%$ & $6,7 \%$ & $53,7 \%$ \\
\hline Géosciences & $48,6 \%$ & $11,5 \%$ & $27,2 \%$ & $63,6 \%$ & $50 \%$ & $40 \%$ & $19,5 \%$ \\
\hline Hydrogéologie & $8,3 \%$ & $5,3 \%$ & $13,2 \%$ & $0 \%$ & $0 \%$ & $0 \%$ & $7,3 \%$ \\
\hline Autres & $13 \%$ & $29,3 \%$ & $24,3 \%$ & $9,1 \%$ & $0 \%$ & $13,3 \%$ & $0 \%$ \\
\hline Total en effectifs & 109 & 113 & 136 & 11 & 6 & 15 & 41 \\
\hline
\end{tabular}

Ce n'est pas tant l'analyse de la bibliographie générale qui est intéressante que la focale sur les seules références scientifiques. Le tableau 1 montre uniquement les proportions de sources scientifiques ${ }^{66}$ de chacun des rapports. Ce qui est tout d'abord remarquable c'est l'importance des citations émanant des Géosciences, de l'Ingénierie et de l'Économie. Les références françaises ciblent quasi exclusivement les documents supportant l'évaluation des ressources (les géosciences représentent entre 19,5\% et 63,6\% des citations), la maîtrise des techniques (l'ingénierie représente jusqu'à $53,7 \%$ des sources) et la rentabilité économique. Fonder la compréhension des enjeux sur le gaz de schiste uniquement sur les géosciences est un choix de cadrage restreint. De plus, le rapport de l'OPECST apparait clairement biaisé, puisqu'il ignore la plupart des études qui contredisent les objectifs de promotion de l'industrie : les auteurs citent par exemple une étude controversée sur les fuites de méthane qui minimise les risques d'émission de gaz à effet de serre tandis qu'ils ignorent les autres études qui démontrent l'importance des fuites. Ils font également appel à des prévisions économiques optimistes produites par des firmes de consultants alors que des économistes universitaires critiquent ces données.

\footnotetext{
${ }^{66}$ Les sources scientifiques sont définies comme étant issues de revues à comité de lecture, d'ouvrages et de rapports scientifiques, et d'études spécifiques commandées dans le cadre de la commission. Elles correspondent majoritairement à des productions de style académiques, les expertises administratives et industrielles sont catégorisées à part. Par ailleurs sont distingués l'hydrogéologie et le reste des géosciences puisque les hydrogéologues tendent à s'opposer aux géologues sur l'évaluation des risques posés par la fracturation hydraulique.
} 
Au Québec, les données scientifiques apparaissent plus diverses. Un plus grand nombre de questions a été posé et une variété de scientifiques issus d'universités, de centres de recherche et de ministères divers se sont penchés sur les multiples aspects de la controverse, notamment grâce aux appels à recherche lancés par l'ÉES. A l'inverse de I'OPECST qui travaille au même moment, l'ÉES met en avant les études controversées sur les fuites de méthane et c'est même un des principaux problèmes soulevés dans le rapport de synthèse : si le Québec développait cette industrie et si les fuites de méthane sont importantes, cela augmenterait radicalement les émissions de gaz à effet de serre de la province. De plus, on voit une place grandissante de la sociologie et de la science politique qui sont mobilisées pour analyser l'acceptabilité sociale, l'état de l'opinion publique ou encore dresser le portrait du mouvement social. Cette évaluation très large est reprise par le BAPE de 2014, et force est de constater l'importance de ces expertises dans les conclusions générales et sur le récit politique qui émerge en 2014 : l'industrie du gaz de schiste est abandonnée par le gouvernement par manque de rentabilité et d'acceptabilité sociale. Alors que l'expertise des sciences sociales est centrale dans le cas québécois, elle est totalement ignorée dans le cas français.

On peut donc déduire que l'origine des références sur lesquelles sont bâties les conclusions des rapports est un élément important du cadrage du problème public. En fonction des expertises mobilisées, l'incertitude légitime à être prise en compte est différente. Le mandat donné aux commissions est la première étape dans ce processus de cadrage. On peut donc suivre B. Wynne ${ }^{67}$ lorsqu'il soutient que peu importe l'objectivité que semble avoir le sens scientifique d'un problème, il est en fait déterminé par des facteurs politiques. L'incertitude sur le gaz de schiste a été gérée par différentes commissions qui ne sont pas toutes scientifiques mais dont l'origine des références détermine à la fois le cadrage du problème et la solution politique. Certaines sont plus scientifiques dans leur démarche (ÉES, CGIET-CGEDD) et réalisent une véritable expertise dans le sens où elles mobilisent des savoirs scientifiques pour l'action. D'autres sont orientées vers le débat public et politique (BAPE, Gonnot-Martin, Havard-Chanteguet) et utilisent l'expertise comme une ressource justificative des discours et des positions politiques. Tandis que l'OPECST réclame une crédibilité scientifique alors qu'il est un dispositif politique, ce qui participe à brouiller les lignes entre expertise scientifique et discours politique. Dans le cas français, les géosciences et l'ingénierie sont perçues comme les plus légitimes pour contribuer à la réduction de l'incertitude. Dans le cas québécois, le cadrage initial est similaire au cadre français, mais le BAPE de 2011 parvient à ouvrir la controverse à une variété d'expertise. Les dynamiques à l'intérieur du forum scientifique sont en lien avec ce qui se déroule dans le forum public. En effet, ce sont les audiences publiques qui déterminent au moins partiellement le contenu des questions de recherche posées durant l'ÉES. En France, les audiences organisées sont sélectives et les membres des commissions vont surtout entendre les représentants de l'industrie (entre 34 et $72 \%$ des individus

\footnotetext{
${ }^{67}$ WYNNE B., op. cit.
} 
auditionnés sont des représentants de l'industrie), ce qui laisse peu d'espace aux revendications des opposants qui sont réduites au seul rejet de la fracturation hydraulique. A rebours du travail de frontières prôné par Collins et Evans, les forums scientifique et public ont des boucles de rétroaction et de renforcement. Ainsi, le cadrage politique de la controverse oriente le type d'expertise mobilisé pour réduire I'incertitude, et cette expertise tend à confirmer en retour la légitimité du cadrage. Aucun organisme ne peut se targuer d'établir le consensus scientifique sur la controverse ${ }^{68}$ dans la mesure où il n'y a pas consensus sur les questions posées. L'information scientifique utilisée pour réduire l'incertitude est donc bien déterminée et cadrée par des facteurs politiques. Il faut aussi noter que l'information scientifique produite n'est efficace que si elle correspond à des objectifs politiques et des significations sociales. Québec a bénéficié de connaissances assez complètes mais cela n'a pas empêché le gouvernement d'autoriser la fracturation hydraulique et d'explorer le pétrole de schiste contre les savoirs mis en avant par l'ÉES. Paris n'avait que des informations partielles mais les décideurs n'ont pu soutenir la production de connaissances à cause de la pression sociale qui a fait du gaz de schiste un tabou politique ${ }^{69}$.

Les dispositifs institutionnels de gestion de la controverse ont donc un rôle majeur dans l'explication des divergences de cadrage du problème public à résoudre. Ils traduisent le récit des acteurs au filtre des choix de mandats, de commissaires d'enquête, de pratiques d'audition et de sélection des sources documentaires. Au Québec, le BAPE est ainsi un contre-pouvoir efficace face à un gouvernement acquis à l'industrie. Ce n'est pas un hasard si à partir de 2015, le ministère de l'Energie et des Ressources Naturelles lance un processus de définition de l'acceptabilité sociale et cherche à mettre en place son propre organisme d'évaluation des projets sur le volet économique. Ce faisant, il cherche à confiner le périmètre d'intervention du BAPE sur les projets énergétiques. En France, malgré des dispositifs institutionnels favorables à l'industrie, la signification sociale et politique du gaz de schiste demeure négative et empêche tout renversement de l'interdiction votée en 2011 sans véritable évaluation et grâce à une forte mobilisation ${ }^{70}$. La situation française semble alors proche du constat fait par C. Restier-Melleray sur l'absence d'un contre-pouvoir académique à l'expertise institutionnelle de l'administration et du parlement. ${ }^{71}$ La consanguinité de l'expertise mobilisée sur le gaz de schiste en France démontre toute la difficulté à mettre en place un organisme indépendant d'évaluation de ce type de projets. Alors qu'au Québec, ce rôle est joué par le BAPE puis par l'EES ad hoc qui fait intervenir une vaste gamme d'experts, en France, les rapporteurs ne peuvent que se désoler du manque d'expertise hors de l'industrie alors même qu'ils participent à confiner l'expertise légitime. L'incertitude et la

\footnotetext{
${ }^{68}$ COLLINS H., EVANS R., Why democracies need science, Cambridge, Polity Press, 2017.

${ }^{69}$ CHATEAURAYNAUD F., ZITTOUN P., " The future they want - or do not want - Shale gas opponents vs. proponents between local motives and global scenarios ", International Conference on Interpretive Policy Analysis, Wageningen University, Netherlands, 2014.

${ }^{70}$ A noter que les déclarations politiques du ministre de la Transition Écologique en juin 2017 vont dans le sens de la fin des permis d'exploration d'hydrocarbures en France, et donc à terme de toute activité d'exploitation.

${ }^{71}$ RESTIER-MELLERAY, op. cit.
} 
manière dont les décideurs la gèrent est en effet en partie déterminée par une dépendance au sentier institutionnel. Le Québec est habitué à recourir au BAPE pour les controverses environnementales et la participation publique sur ces sujets est importante (même si elle ne renverse pas les décisions la plupart du temps). La France est plus centralisée et le parlement souhaite conserver son pouvoir délibératif notamment sur les questions d'énergie comme il l'a fait durant des décennies sur les enjeux nucléaires ${ }^{72}$.

\section{Conclusion: incertitude et action publique}

Face à l'incertitude, les décideurs politiques ont opté pour deux processus différents. En France, les décideurs choisissent de gérer d'abord la mobilisation sociale qui souhaite l'interdiction de la fracturation hydraulique, tout en laissant la porte ouverte à de futures expérimentations. Mais cette seconde phase n'est jamais mise en œuvre à cause de la pression sociale et politique qui empêche toute évaluation de l'industrie et de ses techniques. Ainsi, le cadrage technologique réduit le périmètre de l'incertitude mais celle-ci est finalement utilisée par les opposants pour bloquer la filière depuis 2011. Au Québec, les mobilisations sociales font pression pour une étude d'impact et aboutissent à un dispositif de participation publique et d'évaluation scientifique. Malgré un soutien politique nettement plus important, la filière échoue à s'implanter à cause de son manque de rentabilité mais la technologie - également critiquée - est finalement autorisée pour d'autres projets. Ainsi, le cadrage plus large a permis de réduire l'incertitude sur de nombreux points mais il n'a pas pour autant facilité la validation de toutes les revendications des opposants. Dans les deux cas, les opposants parviennent à faire reconnaître leur revendication principale mais les débordements ${ }^{73}$ ultérieurs sont fort différents.

D'un point de vue comparatif, on note que les dispositifs institutionnels mis en œuvre opèrent des politiques publiques divergentes. La politique française est le produit d'un bricolage dans l'urgence qui court-circuite une évaluation véritable des multiples enjeux de la controverse. Le processus politique est chaotique et le rapport de force se joue dans la mobilisation populaire et l'engagement des élus locaux. Si la politique québécoise est également chaotique, elle engage des procédures plus longues et codifiées. Les multiples rebondissements de la politique sur les hydrocarbures donnent à voir une instrumentalisation des pratiques participatives et la fragmentation du problème. Le gouvernement québécois libéral critique les hydrocarbures au gré de l'évolution du marché mais met en œuvre un cadre règlementaire plutôt ouvert. La comparaison illustre l'ambiguïté de (nouvelles) pratiques de gouvernance - participation, expertise - qui, malgré une égalité procédurale, permettent le maintien d'un cadre favorable à la filière des hydrocarbures

\footnotetext{
72 TOPCU S., La France nucléaire, Paris, Le Seuil, 2013

${ }^{73}$ CALLON M., LASCOUMES P., BARTHE Y, op. cit.
} 
au Québec, et de pratiques (anciennes) de gouvernance - débat parlementaire, mobilisation - qui, malgré une inégalité procédurale, autorisent une avancée pour le mouvement protestataire en France ${ }^{74}$.

\section{Quelles leçons tirer sur l'action publique en situation d'incertitude?}

D’abord, l'incertitude est socialement produite. Elle ne doit pas être considérée comme une donnée neutre par les décideurs politiques. L'incertitude n'est importante que si elle est cadrée comme un problème pour les décideurs politiques. Dans le cas du gaz de schiste, l'incertitude sur la fracturation hydraulique est construite par les opposants qui démontrent les spécificités de la filière et en soulignent les différents risques. La production d'incertitudes est canalisée différemment sur les deux terrains en fonction de la force des acteurs et de leurs discours. Si au Québec, le soutien du gouvernement à l'industrie est initialement une force, la capacité des opposants à valoriser des questionnements alternatifs lors d'audiences publiques permet de rejeter l'industrie sur la base de récits initialement marginaux de reconnaissance de l'inacceptabilité sociale et du manque de rentabilité. A l'inverse, en France, les opposants bénéficient d'un agnosticisme politique qui leur permet d'imposer très rapidement leur définition de la fracturation hydraulique comme un risque inacceptable. Face à l'atonie de l'industrie, leur récit parvient à se transformer en loi sans toutefois s'appuyer sur une réduction scientifique de l'incertitude.

Ensuite, l'incertitude est cadrée politiquement. Les acteurs sont en concurrence pour cadrer le problème de l'incertitude afin de défendre leur agenda. Les dispositifs utilisés pour gérer la controverse ont orienté le cadrage de la controverse. Au Québec, le recours à la participation publique a permis l'ouverture de la controverse à de multiples aspects ignorés en France au niveau institutionnel, puisque les opposants ont seulement revendiqué à travers des manifestations qui ont déformé et simplifié leurs positions. Le processus parlementaire et administratif français a eu tendance à confiner la controverse à un problème de technologie. Au Québec, le cadrage favorise les opposants puisque la filière apparaît inadéquate face aux besoins de la province mais le risque technologique est relativement confiné. ${ }^{75}$

Enfin, l'incertitude est scientifiquement informée. En fonction du cadrage politique, un problème d'incertitude est la plupart du temps géré par un processus de production de connaissances. Les décideurs souhaitent alors à la fois réduire l'incertitude et neutraliser l'information. L'analyse a démontré que le cadrage politique orientait le type d'expertise mobilisé et ce faisant conduisait à une compréhension particulière de la controverse. Pour les décideurs français, l'expertise pertinente et légitime est celle des géologues et des ingénieurs, alors qu'au Québec, les experts mobilisés sont plus divers. C'est un point

\footnotetext{
${ }^{74}$ La France est le premier pays à avoir interdit la fracturation hydraulique, d'autres États avaient seulement instauré des moratoires.

${ }^{75}$ Cette conclusion doit être vue à la lumière des travaux de S. Jasanoff sur les OGM qui soulignent la production d'idiosyncrasies nationales en fonction de l'épistémologie sociale, les règles institutionnelles et les habitudes culturelles. JASANOFF S., 2005, op. cit.
} 
central pour l'analyse de l'incertitude et de l'action publique puisque la façon dont l'incertitude est réduite oriente l'action publique. 